\title{
Open Data
}

Mick Watson

\section{Source}

Mick Watson. (2015). When will 'open science' become simply 'science'? Genome Biol, vol. 16(1).

Open data is the process of releasing both raw and processed data from your experiments, enabling others to analyse it without restriction. 